\title{
Synthesis of Robust Water Reuse Networks Using Fuzzy Nonlinear Programming: Mass Exchange-Based Processes
}

\section{Raymond Girard R. Tan}

Chemical Engineering Department

De La Salle University-Manila

2401 Taft Ave., Manila 1004 PHLIPPINES

Email: tanr_a@dlsu.edu.ph

\section{Dennis E. Cruz}

Industrial Engineering Department

De La Salle University-Manila

2401 Taft Ave., Manila 1004 PHILIPPINES

Email: cruzde@dlsu.edu.ph

\begin{abstract}
Water consumption and effluent generation in industrial plants can be effectively reduced by maximizing utilization of partially contaminated water. A dual approach consisting of graphical pinch methods for targeting followed by the synthesis of water reuse networks using such techniques as mathematical programming is usually employed. Reliable process data is necessary for successful plant retrofitting. In most cases, however, the necessary limiting concentrations and mass loads must be deduced from limited information. It thus becomes necessary to balance the conflicting objectives of minimizing water usage and of ensuring that sufficient stream concentrations fall within their limiting values. The use of fuzzy nonlinear programming for the synthesis of robust water reuse networks is demonstrated using a four-process case study from the literature.
\end{abstract}

Keywords: Fuzzy nonlinear programming, process integration, and water reuse network (WRN).

\section{INTRODUCTION}

Water is used heavily in the process industries for both mass transfer and nonmass transfer operations. The former application includes washing or rinsing of raw materials and process equipment. The latter application includes process cooling and boiler feed, although such uses are not discussed in this work. Examples of such specific water-intensive mass transfer operations are:

- Pulp washing and paper production,
- Surface cleaning or pretreatment in electroplating plants, and

- Clean-in-place of piping and process equipment in food and beverage industries.

Environmental concerns pertaining to fresh water supply sustainability and effluent discharge impacts have resulted in the prevalent use of process integration (PI) to concurrently reduce both plant water requirements and wastewater volume. Specific techniques involving graphical procedures for targeting minimum feasible water usage and different methods for water reuse 
network (WRN) synthesis have evolved from thermal pinch technology, including mathematical programming (Wang and Smith 1994, El-Halwagi 1997, Olesen and Polley 1997, Castro et al. 1999, Hallale 2002, Manan and Foo 2003).

PI projects are either integrated into plant design or implemented through process retrofit. The latter scenario may arise when water supply or effluent treatment capacity suddenly changes. For instance, the limited size of a wastewater treatment plant may make it impossible for the plant to increase production volume and still meet effluent standards. Hence, plant retrofits become necessary to keep the aggregate effluent volume within practical limits. Similarly, the rising costs and unreliable supply of water can provide incentive for the use of process integration techniques. Although reductions in water demand may also be achieved by modifying individual unit operations and processes, an integrated approach reduces the possibility of disrupting operations through changes in product quality.

Nonlinear programming (NLP) models for synthesizing optimal WRN with a fixed number of processes have been formulated (Alva-Argaez et al. 1999, Bagajewicz 2000, Yang et al. 2000). These generic models or superstructures allow multicomponent systems to be handled. Further simplification into linear programming (LP) models is possible for single-component systems by assuming that concentrations of streams entering or exiting individual processes are fixed to the limiting values (Bagajewicz 2000).

In practice, one of the key factors in determining the success of a PI project is the availability of reliable process data to be used for design calculations (Bagajewicz 2000, Tan 2002). Such data is not always obtainable, and actual operations may be subject to considerable noise. Hence, the design of a robust WRN entails explicitly including uncertainty in the model formulation. The methodology used must reconcile two potentially conflicting objectives. On the one hand, water savings can be realized by minimizing water flow through each process. On the other hand, higher water flowrates are desirable to maintain an operating margin of safety when mass loads fluctuate above average levels (Filion et al. 2004, Tan and Cruz 2004).
It should be noted that uncertainty results not just from random variations in operating conditions, but also in the incompleteness of information available to the process designer. For example, limiting concentrations for process streams are not always directly measurable quantities. Often they must be estimated based on solute properties, equipment specifications, or the process engineer's educated guess. This subtle distinction motivates the use of alternative representations of uncertainty in place of traditional probability-based techniques in many applications (Bellmann and Zadeh 1970).

\section{MODEL DEVELOPMENT AND STRUCTURE}

Early work on fuzzy sets in the 1960s quickly led to applications in the field of mathematical optimization. Bellman and Zadeh (1970) provided the foundation for a wide array of optimization tools incorporating fuzzy elements. These have been reviewed by Lai and Hwang (1992) and Rommelfanger (1996).

Fuzzy optimization problems are characterized by objective functions and constraints that are not well-defined in the traditional sense. This concept is illustrated in Figure 1, where the feasible and nonfeasible regions are separated by a fuzzy boundary. In fuzzy mathematical programming, the transition from feasibility to infeasibility occurs gradually across this boundary. By comparison, a sharp boundary separates the feasible and infeasible regions in conventional systems. Once the membership function in the feasible set has been defined, the model can in some cases be reduced to an equivalent crisp version.

The symmetric fuzzy LP (SFLP) method is one such application (Zimmermann 1992). Its key features are as follows:

1. Crisp inequality constraints are converted into fuzzy constraints by introducing tolerances. This change introduces the concept of degree of satisfaction of a constraint, bounded in the interval $[0,1]$.

2. An aspiration level is identified for the objective function. Hence, optimization becomes equivalent to maximizing the degree 
to which the target level is achieved. This degree of satisfaction is also bounded by the interval $[0,1]$. This task is simplified for singlecomponent WRN synthesis since the objective function is automatically bounded by the maximum water flowrate (without reuse) and the theoretical minimum as determined by any targeting technique (Wang and Smith 1994, Castro et al. 1999, Hallale 2002).

3. A new variable, $\alpha$, is added to the LP model. This variable assumes values in the interval $[0,1]$ and serves to concurrently modulate the original objective function and the constraints. The new objective of the SFLP model is to maximize the global degree of satisfaction, $\alpha$, which applies simultaneously to the objective function and the constraints.

Julien (1994) argued that the SFLP model is equivalent to a model with a possibilistic constraint. As a result, the variable $\alpha$ is effectively a numerical reliability index in possibilistic binary state theory of reliability (Cremona and Gao 1997, Tan 2002). Figure 2 shows how the upper half of the mass load possibility distribution defines the failure domain. The logical complement of the failure function is the fuzzy reliability function. The design load must exceed the typical or average load by an appropriate safety margin to achieve a fuzzy reliability level that approaches unity.

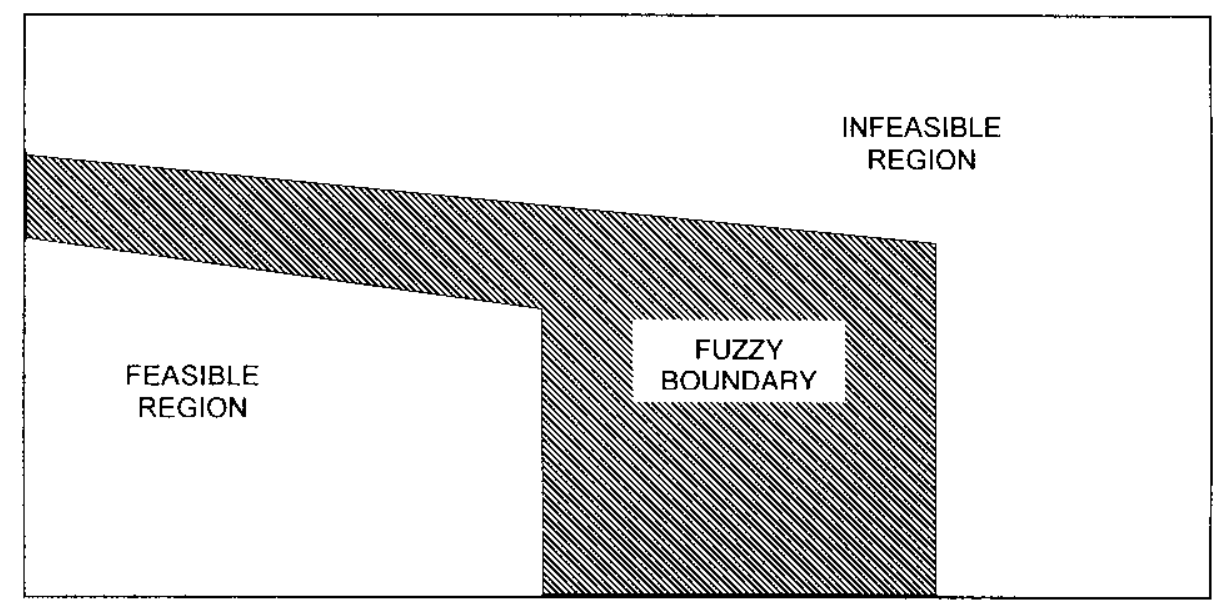

Figure 1. Schematic of Feasible and Infeasible Regions in Fuzzy Mathematical Programming

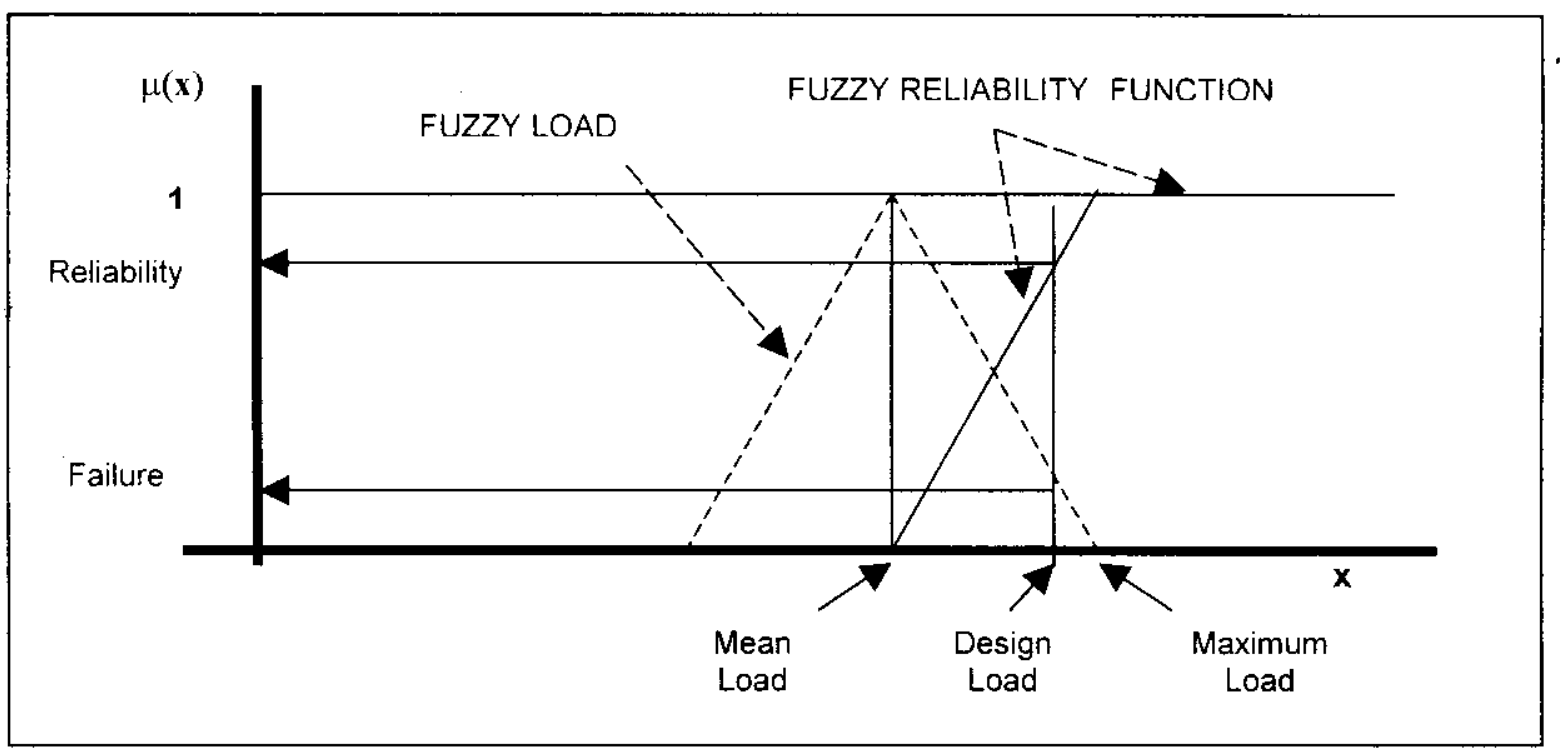

Figure 2. Fuzzy Loads and System Reliability 
In SFLP, the objective function and the constraints are treated identically; hence, the use of the term symmetric. If the degrees of satisfaction are assumed to vary in a linear manner, the SFLP model is very compact, involving only one additional variable and one new (global) objective function to the original problem. Since the SFLP model is just a specially structured LP, it can be solved using the simplex algorithm available in many software packages. It is no longer necessary to convert all constraints to fuzzy form. "Mixed" SFLP models can be treated in the same manner as before.

The use of SFLP in WRN synthesis has previously been demonstrated by Tan and Cruz (2003). The principal limitation of the linear model is that it assumes all streams within the WRN are saturated at their limiting concentrations. This assumption at times leads to suboptimal solutions. This limitation can be overcome by allowing process inlet concentrations to vary as long as they fall within their respective limiting values; however, the resulting model is no longer linear (Alva-Argaez et al. 1999, Bagajewicz 2000, Yang et al. 2000).

The inability of the fuzzy linear model to solve some design problems is addressed by modifying the model to yield a fuzzy nonlinear program (FNLP). The FNLP formulation of the singlecomponent WRN network modeled using the mass exchange unit concept is:

\section{Mathematical model}

\section{Objective function}

$\max \alpha$

Eq. (1) represents a global objective function that is used to reach a compromise between network reliability and water reduction.

\section{Constraints}

$$
\sum_{i} F_{i} \leq F_{\text {tot }}-\alpha\left(F_{\text {tot }}-F_{\text {inin }}\right)
$$

Eq. (2) expresses the relationship between actual water consumption and the absolute minimum requirement of the system without a safety factor. The right hand side is the simplified form of a fuzzy constraining flowrate value
(Zimmermann 1992). The relationship is governed in a linear manner by $\alpha$.

$W_{i}\left(C_{\text {out, max, } i}-C_{i n, i}\right) \geq M_{i}+\alpha\left(\Delta M_{i}\right)$

Eq. (3) expresses the relationship between $\alpha$ and the maximum mass load each process can absorb. The second term on the right hand side represents the safety margin, which, as a consequence, increases the water flow requirement through each process. The entire right hand side is the simplified form of the fuzzy limiting value for mass load (Zimmermann 1992). Note that Eqs. (2) and (3) are inherently conflicting, since the complete satisfaction of the first constraint requires reducing water flow, while complete satisfaction of the second constraint requires increasing water flow to allow dilution of the mass load. A compromise solution is reached by optimizing the variable $\alpha$.

$$
\mathbf{W}_{\mathbf{i}}=\mathbf{F}_{\mathbf{i}}+\sum_{\mathbf{j}} \mathbf{R}_{\mathrm{ji}} \quad \mathbf{i} \neq \mathbf{j}, \forall \mathbf{i}
$$

Eq. (4) gives the total water balance for the stream entering each process. The input stream is a mixture of streams from several sources.

$$
\mathbf{C}_{\mathrm{in}, \mathrm{i}} \mathbf{W}_{\mathrm{i}}=\sum_{\mathrm{j}} \mathbf{C}_{\text {out, } \max , \mathrm{i}} \mathbf{R}_{\mathrm{ii}} \quad \mathrm{i} \neq \mathbf{j}, \forall \mathrm{i}
$$

Eq. (5) is the component balance for input streams into the processes.

$$
\mathbf{W}_{\mathbf{i}}=\mathbf{E}_{\mathbf{i}}+\Sigma_{\mathbf{j}} \mathbf{R}_{\mathrm{ij}} \quad \mathbf{i} \neq \mathbf{j}, \forall \mathbf{i}
$$

Eq. (6) is the total water balance for the splitting of the stream exiting each process. Each of the split streams can then be channeled to different destinations.

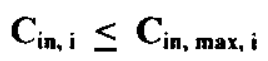

Eq. (7) specifies that the mixed stream entering each process must have a concentration that is within the predefined upper limit for concentration. It is effectively a condition specifying the water quality required for each process.

$$
0 \leq \alpha \leq 1
$$

Eq. (8) specifies the bounds for the value of $\alpha$. 


$$
\mathbf{F}_{\mathrm{i}}, \mathbf{W}_{\mathrm{i}}, \mathbf{R}_{\mathrm{ji}}, \mathbf{E}_{\mathrm{i}} \geq \mathbf{0}
$$

Eq. (9) specifies non-negative values for the system variables.

\section{Parameters}

$C_{\text {out, max }, i}=$ outlet concentration limit of process (i)

$C_{i n, \max , i}=$ inlet concentration limit of process (i)

$F_{\text {tot }} \quad=$ total plant freshwater demand based on maximum mass load assuming no water reuse

$F_{\min }=$ minimum plant freshwater demand as determined by graphical targeting

$M_{i} \quad=$ typical mass load in process (i)

$\Delta M_{\mathrm{i}} \quad=$ mass load tolerance margin in process (i)

\section{Decision Variables}

$C_{i n, i}=$ actual inlet concentration of process (i)
$F_{i} \quad=$ freshwater demand for process (i)

$W_{i} \quad=$ water flowrate through process (i)

$R_{\mathrm{ji}} \quad=$ recycle stream from process $(\mathrm{j})$ to $(\mathrm{i})$

$E_{\mathrm{i}} \quad=$ net effluent of process (i)

$\alpha=$ global degree of satisfaction

\section{CASE STUDY}

The FNLP model is demonstrated using the classic four-process problem of Wang and Smith (1994). This case study is often used as a benchmark problem for the development, testing, and validation of new water integration techniques. Design parameters are shown in Table 1, the only modification being the introduction of fictitious tolerances in the mass loads.

When the mass load tolerances are ignored, it will be seen in Table 2 that the water flowrate for the unintegrated plant (Scenario A) is $112.5 \mathrm{t} / \mathrm{h}$ and that the minimum feasible freshwater flowrate (Scenario C) is $90 \mathrm{t} / \mathrm{h}$. The latter is easily determined using any of the traditional graphical targeting methods. Figure 3 shows

Table 1. Design Parameters for Case Study (Adapted from Wang and Smith 1994)

\begin{tabular}{|c|c|c|c|c|}
\hline \multirow{2}{*}{ Process } & \multicolumn{2}{|c|}{ Limiting Concentrations $(\boldsymbol{m g} / \mathbf{l})$} & \multicolumn{2}{c|}{ Mass Load $(\boldsymbol{k g} / \boldsymbol{h})$} \\
\cline { 2 - 5 } & $\boldsymbol{C}_{\boldsymbol{i n}}$ & $\boldsymbol{C}_{\text {out }}$ & $\boldsymbol{M}$ & $\boldsymbol{M}+\boldsymbol{\Delta M}$ \\
\hline 1 & 0 & 100 & 2.00 & 2.20 \\
\hline 2 & 50 & 100 & 5.00 & 5.50 \\
\hline 3 & 50 & 800 & 30.00 & 38.00 \\
\hline 4 & 400 & 800 & 4.00 & 5.00 \\
\hline
\end{tabular}

Table 2. Water Flowrates and Savings of Different Reuse Scenarios

\begin{tabular}{|c|l|c|c|}
\hline Scenario & \multicolumn{1}{|c|}{ Description } & \multicolumn{1}{|c|}{$\begin{array}{c}\text { Flowrate } \\
(\boldsymbol{t} / \boldsymbol{h})\end{array}$} & $\begin{array}{c}\text { Relative Change } \\
(\boldsymbol{\%})\end{array}$ \\
\hline A & No reuse based on typical mass load & 112.5 & 0 \\
\hline B & $\begin{array}{l}\text { No reuse based on maximum possible } \\
\text { mass load }\end{array}$ & 130.8 & +16.2 \\
\hline C & Non-robust network & 90.0 & -20.0 \\
\hline D & Robust network & 99.5 & -11.6 \\
\hline
\end{tabular}




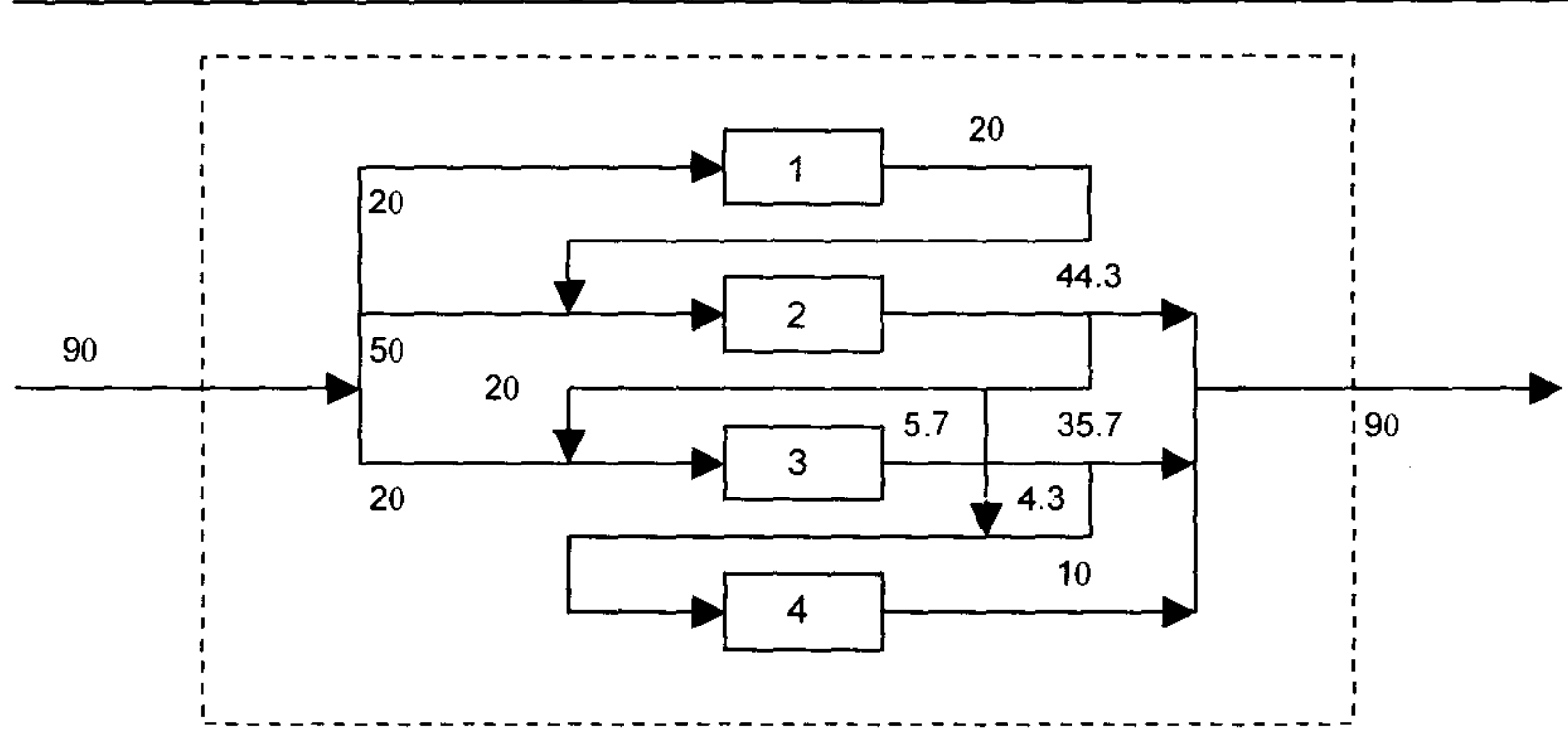

Figure 3. Nonrobust Water Reuse Network (Indicated Flowrates in $t / h$ )

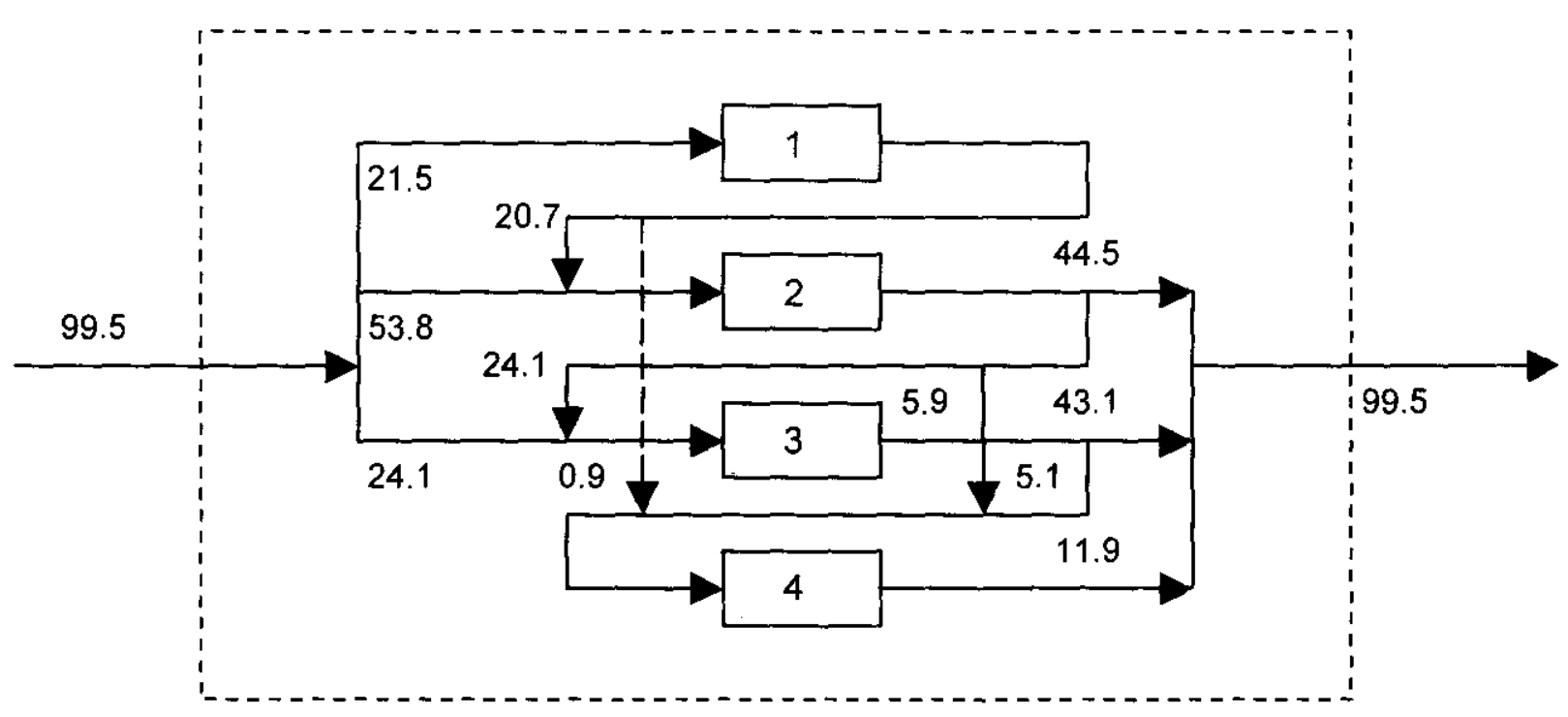

Figure 4. Robust Water Reuse Network (Indicated Flowrates in $t / h$ )

a network layout that achieves this target value. Although this differs from the solution proposed by Wang and Smith (1994) both are in fact feasible. All networks based on this $90 \mathrm{t} / \mathrm{h}$ target flowrate will fail once the typical or average mass loads are exceeded during operation. When network failure is manifested by stream concentrations exceeding their respective limits, the solvent will be unable to properly perform its cleansing function within the processes. In practice this will result in the generation of substandard products.
If the maximum possible mass loads are used, the water demand for the unintegrated plant (Scenario B) will be $130.8 \mathrm{t} / \mathrm{h}$, or $16.2 \%$ more than the baseline flowrate. The problem is thus to design a network that approaches the minimum feasible flowrate while simultaneously providing a safety factor to allow for uncertainty in the mass load estimates. However, it is unclear exactly how to apply safety factors because the processes are interconnected in such a way that excess concentrations can propagate through the network (Tan 2002). FNLP allows the WRN configuration 
to be determined while simultaneously relaxing the design to allow for possible variations in the mass load. The resulting robust network is shown in Figure 4. Except for the reuse of $0.9 \mathrm{t} / \mathrm{h}$ of discharge from Process 1 into Process 4 (broken line), the interconnections are almost identical to those found in the nonrobust network with somewhat greater flowrates to allow for more dilution. The total fresh water demand-and effluent generation-is $99.5 \mathrm{t} / \mathrm{h}$ (Scenario D), which is still $11.6 \%$ less than the demand of the unintegrated plant (Scenario A). While the water requirement of this network is about $10 \%$ greater than that of the conventional one, it is more able to withstand mass load fluctuations without exceeding the limiting stream concentrations of each process.

The optimal value of $\alpha$ is found to be 0.768 . For each process, the maximum mass load that can be tolerated without exceeding concentration limits is $M+\alpha \Delta M$; numerical values are shown in Table 3. If Processes 1-4 simultaneously operate at mass loads of 2.15 , $5.38,36.14$, and $4.77 \mathrm{~kg} / \mathrm{h}$, respectively, all water streams will be at their limiting concentrations. Table 4 shows the corresponding stream concentrations when operating at the average expected mass loads of $2,5,30$, and $4 \mathrm{~kg} / \mathrm{h}$. Stream concentrations for the nonrobust network are at the design limits, except for the inlet stream of Process 2. It is evident that this network will fail once mass loads fluctuate above the mean values. By comparison, the stream concentrations of the robust network are below the limiting values, providing some margin of safety should mass loads vary during operation.

\section{CONCLUSIONS}

Use of fuzzy nonlinear programming for the synthesis of robust water reuse networks has been demonstrated using a four-process case study. This method provides a means of balancing water reduction targets with the need to meet process limiting concentrations. This compromise arises from uncertainties about the true mass loads encountered during operation.

Further work on the use of fuzzy mathematical programming and related techniques in the design of water reuse networks will focus on the modeling of the following cases:

- Multicomponent systems,

- Water reuse networks with regeneration,

- Application to hydrogen and property pinch problems, and

- Use of expert systems for network synthesis.

Table 3. Maximum Tolerable Mass Loads

\begin{tabular}{|c|c|c|c|}
\hline \multirow{2}{*}{ Process } & \multicolumn{3}{|c|}{ Mass Load $(\mathbf{k g} / \boldsymbol{h})$} \\
\cline { 2 - 4 } & $\boldsymbol{M}$ & $\boldsymbol{M}+\Delta \boldsymbol{M}$ & $\mathbf{M}+\alpha \Delta \boldsymbol{M}$ \\
\hline $\mathrm{l}$ & 2.00 & 2.20 & 2.15 \\
\hline 2 & 5.00 & 5.50 & 5.38 \\
\hline 3 & 30.00 & 38.00 & 36.14 \\
\hline 4 & 4.00 & 5.00 & 4.77 \\
\hline
\end{tabular}

Table 4. Stream Concentrations at Average Mass Load Levels

\begin{tabular}{|c|c|c|c|c|}
\hline \multirow{2}{*}{\multicolumn{2}{|c|}{ Process Stream }} & \multicolumn{3}{|c|}{ Concentration $(m g / l)$} \\
\hline & & \multirow{2}{*}{$\begin{array}{r}\begin{array}{r}\text { Design } \\
\text { Limit }\end{array} \\
0\end{array}$} & \multirow{2}{*}{$\begin{array}{c}\begin{array}{c}\text { Nonrobust } \\
\text { Network }\end{array} \\
0\end{array}$} & \multirow{2}{*}{$\begin{array}{c}\begin{array}{c}\text { Robust } \\
\text { Network }\end{array} \\
0\end{array}$} \\
\hline \multirow{4}{*}{ Process Inlets } & 1 & & & \\
\hline & 2 & 50 & 30 & 26 \\
\hline & 3 & 50 & 50 & 46 \\
\hline & 4 & 400 & 400 & 340 \\
\hline \multirow{4}{*}{ Process Outlets } & 1 & 100 & 100 & 93 \\
\hline & 2 & 100 & 100 & 93 \\
\hline & 3 & 800 & 800 & 669 \\
\hline & 4 & 800 & 800 & 676 \\
\hline
\end{tabular}




\section{REFERENCES}

Alva-Argaez, A., Vallianatos, A., and Kokossis, A. (1999). "A multi-contaminant transhipment model for mass exchange networks and wastewater minimisation problems," Comput. Chem. Eng., 23, 1439-1453.

Bagajewicz, M. ( 2000). "A review of recent design procedures for water networks in refineries and process plants," Comput. Chem. Eng., 24, 2093-2113.

Bellmann, R. E., and Zadeh, L. A. (1970). "Decision-making in a fuzzy environment," Manage. Sci., 17, 141-164.

Castro, P., Matos, H., Fernandes, M. C., and Pedro Nunes, C. (1999). "Improvements for mass-exchange networks design," Chem. Eng. Sci., 54, 1649-1665.

Cremona, C., and Gao, Y. (1997). "The possibilistic reliability theory: Theoretical aspects and applications," Struct. Saf., 19, 173-201.

El-Halwagi, M. M. (1997). Pollution prevention through process integration: Systematic design tools, Academic Press, New York.

Filion, Y. R., Karney, B. W., and Adams, B. J. (2004). "Multiobjective design of water networks with random loads," Environmental Informatics Archives, 2, 252-257.

Hallale, N. (2002). "A new graphical targeting method for wastewater minimisation," Adv. Environ. Res., 6, 377-390.

Julien, B. 1994. "An extension to possibilistic linear programming," Fuzzy Set. Syst., 64, 195-206.

Lai, Y. J., and Hwang, C. L. (1992). Fuzzy mathematical programming: Methods and applications, Lecture notes in economics and mathematical systems, Berlin, Springer-Verlag.

Manan, Z. A., and Foo, C. Y. (2003). "Setting targets for water and hydrogen networks using cascade analysis." American Institute of Chemical Engineers Annual Meeting, San Francisco CA.

Olesen, S. G., and Polley, G. T. (1997). "A simple methodology for the design of water networks handling single contaminants," Trans. I. Chem. E., Part A, 75, 420-426.
Rommelfanger, H. (1996). "Fuzzy linear programming and applications," Eur. $J$. Oper. Res., 92, 512-517.

Tan, R. R. (2002). "Assessing the sensitivity of wastewater reuse networks to noisy loads using possibility theory." Proceedings of the 2002 Chemical Engineering Congress, Manila, Philippines.

Tan, R. R., and Cruz, D. E. (2003). "Synthesis of robust water reuse networks using symmetric fuzzy LP superstructures." Proceedings of the Interinational Conference on Chemical and Bioprocess Engineering, Kota Kinabalu, Malaysia.

Tan, R. R., and Cruz, D. E. (2004). "Synthesis of robust water reuse networks for singlecomponent retrofit problems using symmetric fuzzy linear programming," Comput. Chem. Eng., 28, 2547-2551.

Wang, Y. P., and Smith, R. (1994). "Wastewater minimization," Chem. Eng. Sci., 49, 9811006.

Yang, Y. H., Lou, H. H., and Huang, Y. L. (2000). "Synthesis of an optimal wastewater reuse network," Waste Manage., 20, 311-319.

Zimmermann, H. J. (1992). "Methods and applications of fuzzy mathematical programming." In: Yager, R. R., and Zadeh, L. A., eds., An introduction to fuzzy logic applications in intelligent systems, Boston, Kluwer. 